\title{
Shape Transformations During the Growth of Gold Nanostructures
}

\author{
N Ahmad ${ }^{1}$, Y Le Bouar ${ }^{2}$, C Ricolleau ${ }^{1}$ and D Alloyeau ${ }^{1}$ \\ ${ }^{1 .}$ Laboratoire Matériaux et Phénomènes Quantiques, Université Paris 7 - CNRS, Paris, France. \\ 2. Laboratoire d'Etude des Microstructures, ONERA /CNRS, B.P. 72, 92322 Chatillon, France.
}

Liquid cell transmission electron microscopy (LCTEM) has rapidly emerged as a potent tool for understanding the dynamical processes taking place at solid / liquid interfaces. Imaging colloidal solutions with the high temporal and spatial resolutions of TEM enables understanding the growth mechanisms that control the final size and morphology of nanoparticles. Nevertheless, conclusive LCTEM experiments require understanding the effects of electron-irradiation on the nanoscale phenomena under study. Radiolytic syntheses driven by the electron beam were performed in this work to study the effects of the dose history (including the instantaneous dose rate and the cumulative dose) and the solvent nature on the shape of gold nanoparticles. The straightforward control over the concentration of reducing agents (radiolytically-produced hydrated or solvated electrons) provides mechanistic insights on the growth of highly desired nanocrystal shapes for plasmonic applications.

Liquid cell experiments were carried out on an aberration corrected JEOL ARM 200F operated at $200 \mathrm{KV}$, by using a commercial liquid-cell holder provided by Protochips Inc. $1 \mathrm{mM} \mathrm{HAuCl} \mathrm{H}_{4}$ in water or methanol was analyzed in a $150 \mathrm{~nm}$-spacer liquid cell. Growth experiments were conducted under two extreme regimes of dose rate (over 150 electrons $/ \AA^{2}$ s and below 1 electrons $/ \AA^{2}$ s) in both TEM and STEM modes.

Under high dose rate we observed the growth of dendritic nanostructures (Fig. 1a). By comparing LCTEM observations with an extended diffusion-limited aggregation model (Fig. 1b), we explicitly reveal the molecular and atomic diffusion processes that impact the shape of these dendritic nanostructures [1]. Besides the well-established link between the dose rate and the growth speed of the nanostructures [2,3], we have demonstrated that the cumulative dose in the irradiated area can also induce drastic transitions in the growth mode of the nanostructures. For instance, high dose rate observation severely affects the concentration of precursors in and around the irradiated area, resulting in the formation of anisotropic tree-like structures over spherical nanoparticles (Fig. 1a).

Under low dose rate, reaction-limited growth leads to the formation of highly facetted nanoclusters. The growth is then dominated by thermodynamic effects, because the lower adsorption rate of gold atoms provides enough time for the clusters to reach an equilibrium shape that depend on intrinsic and extrinsic parameters [3]. We show that crystal defects (intrinsic parameter) or the nature of the solvent that modulates the surface energies of crystal facets (extrinsic parameters) can both drive shape transformations during the growth of the nanoparticles. Remarkably, we reveal the formation mechanisms of highly symmetric 2D and 3D nanostars enclosed by high-index facets. These in situ studies could help in designing new seed-mediated methods or capping strategies to fabricate metallic nanostars.

References:

[1] N Ahmad et al, Adv Struct Chem Imag 2 (2016). 
[2] T Woehl et al, ACS Nano 10 (2012), pp. 8599-8610.

[3] D Alloyeau et al, Nanoletters 15 (2015), pp. 2574-2581.

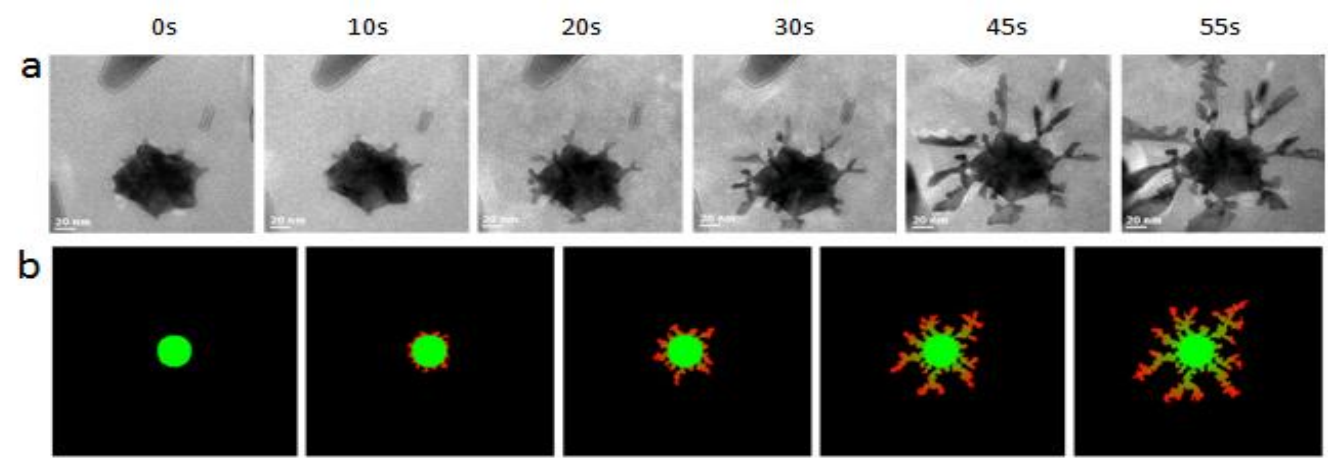

Figure 1: High dose rate liquid TEM experiments. (a) Growth of sharp dendrites over spherical nanoparticle. Spherical nanoparticles are firstly formed in pristine area of the liquid cell with homogenous precursor concentration, followed by a dendritic growth starting at $\mathrm{t}=0 \mathrm{~s}$. This transition in the growth mechanisms is due to a precursor depletion in the irradiated area. (b) Shape transition observed experimentally reproduced with an extended diffusion-limited-aggregation model.
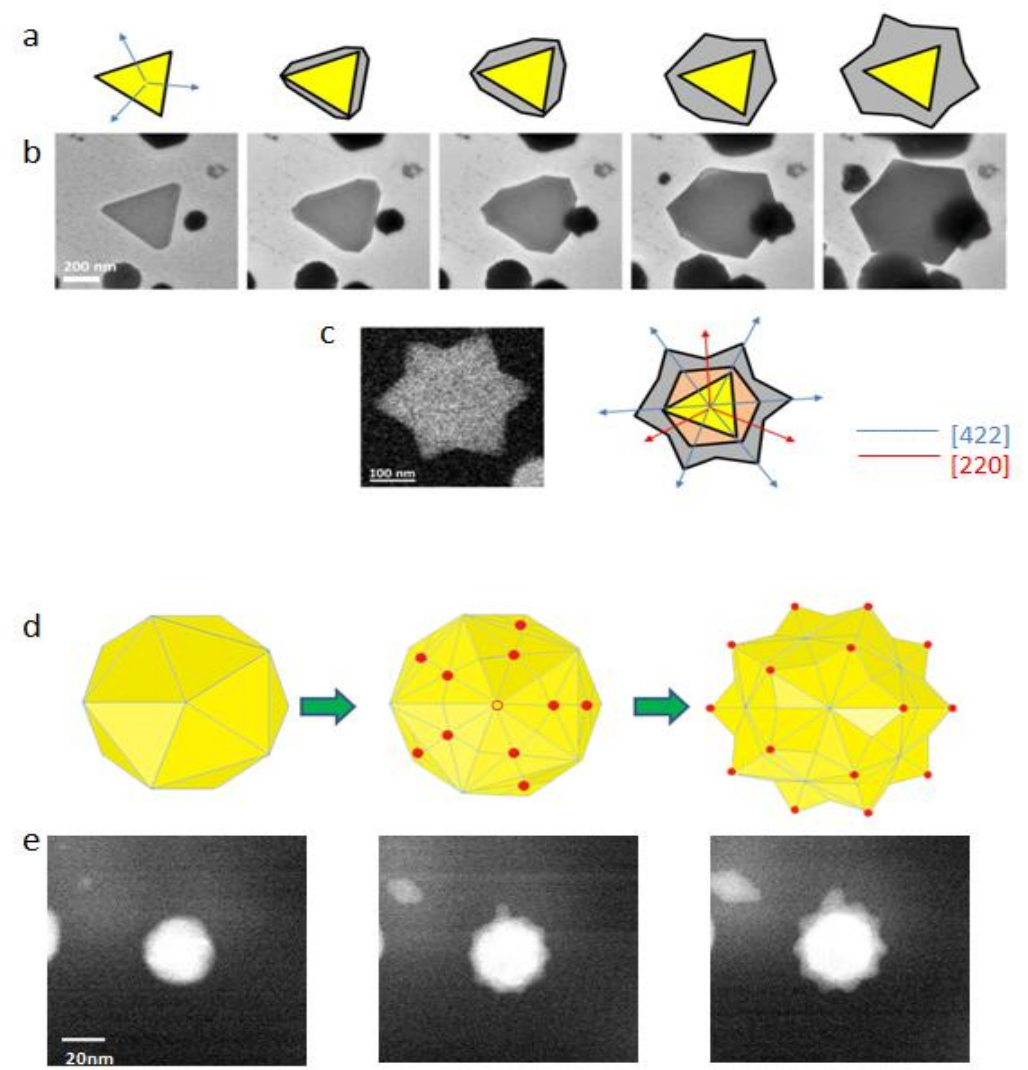

Figure 2: (a) Geometric model showing formation of a 2D gold nanostar from a 2D gold nanoprism through a continuous growth along the [422] directions. (b) In-situ TEM observations of the transformation process. (c) In-situ STEM image of a well faceted 2D nanostar. (d) Formation mechanism of a gold nanostar from an icosahedral gold seed crystal. (e) In-situ STEM images of various stages of growth of 3D nanostar. 